Revue

Revue de l'histoire des religions

de Ihistoire des religions

1 | 2016

Varia

\title{
Philippe BORGEAUD et Doralice FABIANO (dir.), Perception et construction du divin dans l'Antiquité
}

Genève, Droz (« Recherches et Rencontres », 31), 2013

Sarah Rey

\section{CpenEdition}

Journals

Édition électronique

URL : http://journals.openedition.org/rhr/8493

DOI : 10.4000/rhr.8493

ISSN : 2105-2573

Éditeur

Armand Colin

Édition imprimée

Date de publication : 1 mars 2016

Pagination : 101-104

ISBN : 978-2-200-93059-2

ISSN : 0035-1423

Référence électronique

Sarah Rey, «Philippe borgeaud et Doralice fabiano (dir.), Perception et construction du divin dans l'Antiquité », Revue de l'histoire des religions [En ligne], 1 | 2016, mis en ligne le 04 avril 2016, consulté le 22 septembre 2020. URL : http://journals.openedition.org/rhr/8493; DOI : https://doi.org/10.4000/rhr 8493

Ce document a été généré automatiquement le 22 septembre 2020.

Tous droits réservés 


\section{Philippe BORGEAUD et Doralice FABIANO (dir.), Perception et construction du divin dans l'Antiquité}

Genève, Droz («Recherches et Rencontres », 31), 2013

\section{Sarah Rey}

\section{RÉFÉRENCE}

Philippe BORGEAUD et Doralice FABIANo (dir.), Perception et construction du divin dans

l'Antiquité, Genève, Droz (« Recherches et Rencontres », 31), 2013, 22 cm, 354 p., 49,58 €,

ISBN 978-2-600-01644-5.

1 Ce volume est issu du colloque «Perception des dieux, émotions, maîtrise rituelle : corps divins, corps humains » (Genève, 24 au 26 mars 2011), titre indiquant assez la complexité de l'objectif assigné à chaque participant. Comment la divinité et les dévots entrent-ils en contact sensible? Douze articles d'une grande variété tentent d'y répondre : les sources iconographiques ou archéologiques côtoient les textes littéraires et les inscriptions; la Grèce classique et hellénistique, la Mésopotamie, la Phénicie, la Rome républicaine et impériale, et même la Crète minoenne suscitent une série de réflexions relevant des Affective Sciences (les directeurs de l'ouvrage appartiennent tous deux au Swiss Center for Affective Sciences).

2 L'introduction de Ph. Borgeaud et D. Fabiano vient poser les cadres de l'analyse : « les dieux sont des êtres de métamorphose, en métamorphose. S'ils ont un corps, ils ne sont pas limités à un corps ». Telle qu'elle est rapportée par Callimaque, l'histoire édifiante de l'Hermès Perpheraîos d'Ainos en Thrace, pris dans des filets de pêche, brûlé sans succès par des pêcheurs impies, rejeté à la mer, pêché une nouvelle fois, montre comment une représentation divine finit par s'imposer comme un objet sacré : le dieu surgit à l'improviste dans le quotidien des hommes et fait sentir sa grandeur par son essence supérieure, sa supra-matérialité, qui porte à interroger sur sa fabrication. Dès 
lors, les techniques des peintres, sculpteurs, céramistes, architectes entrent inévitablement dans le propos des auteurs, car ces hommes de l'art concourent à donner une forme aux dieux: "l'image cultuelle est trompeuse certes, mais indéniablement efficace »(p.9).

3 En ouverture, Annie Dubourdieu propose une réflexion sur l'originalité romaine en matière d'apparitions divines.

4 Il n'y a pas de terme latin pour dire l'épiphanie, pourtant les divinités ne manquent pas de se manifester aux Romains. Ainsi, les Dioscures sont deux entités divines qui se donnent à voir au lac Régille, en 496 av. J.-C., et sur la route de Réate à Rome, en 168 av. J.-C. Mais c'est plutôt par la statuaire que les Romains entrent en dialogue visuel avec les dieux. A. Dubourdieu envisage alors la richesse sémantique des termes signum et simulacrum, les deux mots les plus utilisés pour évoquer les représentations divines. Par la suite, Corinne Bonnet et Adeline Grand-Clément s'attachent à suivre deux errances divines pan-méditerranéennes: celle de l'Apollon de Géla et celle de l'Artémis de Ségeste, qui font les frais de godnappings successifs, reflets de tensions géopolitiques. Le premier est transféré de force à Tyr et finalement libéré par Alexandre le Grand en 332 av. J.-C.; la seconde - plus ballottée encore - est établie à Carthage jusqu'à la destruction de la ville en 146 av. J.-C., puis regagne Ségeste avant que Verrès ne fasse main basse sur elle. Les deux récits exemplaires illustrent la puissance des statues qui servent à tracer une frontière entre la juste piété et la superstition, démarcation aux connotations ethniques : les barbares - les Carthaginois et les Phéniciens - sont ceux que les dieux désertent.

Hélène Collard s'intéresse, quant à elle, à la trentaine de vases qui figurent un dieu et sa propre effigie dans un dédoublement très signifiant. Le vol des palladia permet, de la sorte, la représentation d'Athéna "en personne» se plaçant entre Diomède et Archimède, tandis que les deux héros tiennent chacun une statuette de la déesse. Le viol de Cassandre fait apparaître la même divinité, scindée entre sa propre individualité et sa représentation. D'autres scènes tirées du cycle troyen incitent à matérialiser cette partition qui n'est pas une "simple redondance" : l'imagier peint les immortels en action, au milieu des hommes ou non loin d'eux. À chaque fois, la statue est un medium efficace, mais n'est pas le strict équivalent de la divinité. En s'attardant sur l'iconographie et la piété des artisans en Grèce ancienne, Anne-Catherine Gillis fait place à une imagerie de la difformité et de la laideur, qu'on a longtemps rattachée à la marginalité des métiers artisanaux (en accord avec le personnage d'Héphaïstos dans les textes homériques), avant de lui prêter une autre symbolique. L'artisan n'est-il pas à sa mesure un daimon, capable du pire comme du meilleur? N'entretient-il pas un rapport étroit avec le feu, cet instrument de civilisation et de destruction?

6 Sophie Montel se penche, elle, sur les scénographies architecturales dans les sanctuaires et les espaces publics grecs. Cette analyse court sur le temps long, du site minoen d'Anémospilia jusqu'au temple hellénistique de Despoina à Lycosoura, en passant par le Dionysion de Thasos. Il ressort de cette large mise en perspective que les statues de divinité sont rendues visibles par plusieurs canaux, notamment par des effets de miroirs soigneusement calculés. C'est aussi ce versant optique de la communication entre les dieux et les hommes, couplé au toucher et à l'audition, qui occupe Lorenz Baumer, lequel s'arrête sur le cas des sanctuaires d'Asclépios, lieux d'intenses émotions collectives. Lors des incubations, le dieu touche les malades et se fait entendre (il siffle dans le Ploutos d'Aristophane). Tantôt il défend qu'on le voie, 
comme en témoigne un épisode des Iamata d'Epidaure, tantôt il offre des «perspectives » divines : la structure des abata permet des coups d'œil suggestifs sur le centre des Asclépieia. Il est encore question des effets physiques de la divinité, avec l'étude de Doralice Fabiano consacrée à la nympholepsie. Cette dernière survient souvent dans un lieu enchanteur, où la végétation et les cours d'eaux favorisent la perte de conscience. Celui qui est saisi par les nymphes ne s'appartient plus, il peut trouver dans cet état second la matière de discours sans fin, parfois prophétiques. Mais le nympholepte, exalté bucolique, n'est pas que le jouet des nymphes, il tient également un rôle créateur dans la délimitation d'espaces cultuels dédiés à celles qui l'inspirent. La dimension acoustique de la présence divine est ensuite au cœur de l'article d'AnneCaroline Rendu Loisel, qui trouve dans les textes cunéiformes akkadiens et sumériens la mention de présages liés au tonnerre dont les bruits varient selon de subtiles distinctions: le grand dieu Adad peut tonner comme un dragon, comme une souris, comme un lion, etc. À chaque bruit sa prédiction, toujours effrayante. Dans un autre contexte, lorsqu'on cherche à exorciser un malade, un instrument à percussion vient chasser le démon. Et seules les paroles murmurées d'un officiant ont le pouvoir de sacraliser un objet de culte. En définitive, la religion de la Mésopotamie ancienne accorde une grande importance aux sons, vecteurs de sacré.

7 Athanassia Zografou décrit les curieux procédés de "programmation " des rêves d'après les Papyri Graecae Magicae. Selon ces papyrus de l'Antiquité tardive, laurier et armoise sont deux plantes qui faciliteraient les visions fantastiques. Quant aux lampes qui jouxtent la tête des dormeurs, elles sont utilisées comme des oniro-transmetteurs en quelque sorte, des sièges en miniature du divin, « un vrai sanctuaire de poche » selon la belle formule de l'auteur.

8 Stéphanie Paul se concentre sur un matériel épigraphique mettant en scène les Artémis d'Asie Mineure, déesses qui n'hésitent pas à se manifester lorsque leurs cités demandent un soutien divin. Les autorités de Magnésie du Méandre, de Cnide ou de Bargylia en Carie prennent acte de ces épiphanies qui se produisent à point nommé. Elles accroissent les honneurs rendus à la déesse, qui confirme sa position hégémonique (faut-il parler de " divinité tutélaire » ?) dans les panthéons locaux.

9 Marie-Christine Villanueva-Puig reprend le dossier du ménadisme, phénomène par lequel un individu (féminin) se laisse posséder par Dionysos aux multiples formes d'apparition: jeune créature aux traits asexués ou homme d'âge mûr, lumière inquiétante ou bruit assourdissant. Le dieu s'empare des corps des ménades et atteint d'autres corps : ceux des enfants saisis sans égard par les ménades ou ceux des animaux déchiquetés avec violence.

10 Enfin, Anne-Françoise Jaccottet examine les modalités de l'apothéose (ou consecratio) dont bénéficient certains empereurs et impératrices. Ces grands hommes et grandes femmes ne quittent leurs corps qu'à grand-peine : on aperçoit leurs fantômes (parfois au prix de faux témoignages), on brûle leurs bustes et, finalement, les sculpteurs les représentent en voie de divinisation sous leur apparence familière.

11 L'ensemble de ces contributions enrichit beaucoup le questionnaire relatif à la perméabilité des sphères divine et humaine. Grâce à ces divers travaux, nous comprenons mieux comment les corps des dieux et des fidèles entrent en correspondance par un ensemble de dispositifs sensoriels, où domine la composante visuelle. Il apparaît en outre qu'aucune émotion religieuse n'est indépendante de son contexte politique, géographique et culturel. 


\section{AUTEURS}

SARAH REY

Université de Valenciennes et du Hainaut Cambrésis. 